\title{
Emodialisi e sostenibilità ambientale
}

\author{
Elena Mancini \\ U.O. Nefrologia Dialisi Ipertensione, Azienda Ospedaliero-Universitaria Policlinico S. Orsola, Bologna - Italia
}

\begin{abstract}
Hemodialysis and environmental sustainability
The healthcare system is a great producer of greenhouse gases, and user of natural resources as well. Any dialysisrelated activity, from procurement of materials to organization of patients' transport, from administration of dialysis therapy itself to inevitable production of large amounts of waste, makes dialysis one of the health activities using most natural resources, indirectly producing greenhouse emissions.

Water consumption is, inevitably, the factor with the highest environmental impact. We, the nephrologists, are generally not well informed on the topic, and few of us know the actual water requirement per single dialysis treatment. Particularly, little is known about the large volume of water wasted with reverse osmosis: despite the industry's efforts to reutilize wasted water, currently some $30 \%$ of the water used by reverse osmosis is still lost. However, in some countries modified water systems already exist, recovering and reusing water lost for different purposes, such as cleaning, steam production and gardening.

The power requirement, waste production and even the design of dialysis centers are further issues in the dialysisecocompatibility relationship. Awareness of these issues is surely the first step. It is now vital that we keep ourselves informed and updated, and raise these issues in our dialysis centers. English and Australian experience will hopefully pave the way for certain small, already feasible acts. In the future, the immediate future, the design of dialysis centers and especially of water treatment systems is bound to undergo huge changes if we are to reduce the negative impact on the environment.
\end{abstract}

Keywords: Dialysis, Ecocompatibility, Environment, Sustainability

\section{Introduzione}

"Carbon footprint", letteralmente "impronta di carbonio", è una sorta di indice surrogato per quantificare le emissioni di anidride carbonica nell'aria, coinvolta nella produzione dei gas serra, a cui si attribuisce il fenomeno dell'aumento della temperatura media globale. La mente va subito ai tanti servizi, articoli, approfondimenti, che tutti sentiamo e leggiamo, sull'argomento dei cambiamenti climatici, che sono stati considerati la più grande sfida alla salute globale del $21^{\circ}$ secolo.

Does this matter with nephrology, with dialysis? Yes, a lot.

Received: May 19, 2020

Accepted: June 10, 2020

Published online: July 17, 2020

Indirizzo per la corrispondenza:

Elena Mancini

U.O. Nefrologia Dialisi Ipertensione

Azienda Ospedaliero-Universitaria Policlinico S. Orsola

Via P. Palagi 9

40138 Bologna - Italia

elena.mancini@aosp.bo.it
I sistemi sanitari contribuiscono in modo pesante all'emissione di gas serra e al consumo di risorse naturali. Chi si è dedicato a queste analisi ha ricavato che il programma di dialisi cronica è, in termini di consumo di risorse e di generazione di rifiuti, al primo posto nella produzione annuale di gas fra le attività sanitarie. Uno studio australiano ha dimostrato già anni fa che il footprint annuale di un paziente in emodialisi cronica in un centro satellite australiano era pari a 10,2 t $\mathrm{CO}_{2}$-eq (tonnellate equivalenti di $\mathrm{CO}_{2}$ ), pari a oltre la metà della produzione annuale per singolo cittadino di $\mathrm{CO}_{2}$, stimata a $18,8 \mathrm{t}$ $\mathrm{CO}_{2}$-eq. Sempre in Australia, è stato calcolato che un centro dialisi periferico in Geelong contribuiva alla produzione di gas serra in maniera equivalente alla metà dell'emissione di gas serra creata da una città media australiana (1-4).

Questi esempi provano inequivocabilmente che, anche nel mondo della dialisi, bisogna cominciare a pensare in termini di "sostenibilità". La British Renal Society e la British Renal Association hanno introdotto la sostenibilità come settima dimensione della qualità dell'assistenza, insieme a sicurezza, tempestività, efficacia, efficienza, equità e percezione del paziente (5). In generale, una medicina sostenibile è quella che va incontro ai bisogni attuali delle persone, senza, però, compromettere le possibilità delle future generazioni di poter assicurare i loro bisogni, quindi implica l'impegno a non depredare 
le risorse naturali e a mantenere un equilibrio ecologico. Per questi obiettivi, bisogna pensare a come ridurre l'emissione diretta e indiretta di gas serra da parte del mondo della dialisi e, più in generale, a come ridurre il consumo delle risorse naturali.

\section{Emodialisi e impatto ambientale}

Sono vari i motivi di spesa, generatori, inevitabilmente, di "inquinamento" o di consumo di risorse naturali da parte di un centro dialisi: consumo di acqua ovviamente al primo posto, consumo di energia elettrica, approvvigionamento di materiali, produzione e gestione dei rifiuti, le modalità di costruzione stesse.

L'acqua è il più facile esempio di risorsa naturale finita, come le foreste, le risorse minerarie, la terra, i gas naturali. Quanti di noi guardano, ogni tanto, il contatore presente nei locali sede dell'impianto dell'acqua per vedere il consumo giornaliero di acqua? Un centro dialisi come il nostro, dove si eseguono circa 50 dialisi al giorno, consuma ogni giorno $21 \mathrm{~m}^{3}$ di acqua, cioè 21.000 litri. Di questi, circa $8 \mathrm{~m}^{3}$ sono utilizzati per il pre-trattamento e $13 \mathrm{~m}^{3}$ vanno al circuito e alle macchine. $\mathrm{Ma}$, inevitabilmente, una parte considerevole di acqua viene persa. Questo nonostante l'industria abbia già da tempo instaurato dei meccanismi di recupero dell'acqua inutilizzata nello stesso impianto (cosiddetto permeato):

1) un sistema di ricircolo interno, per cui il permeato non utilizzato viene recuperato e rientra nell'osmosi;

2) un meccanismo a valvola presente sullo scarico, con apertura periodica, che regola proporzionalmente il volume di scarico in base al reale consumo dei monitor.

Malgrado ciò, il $\mathbf{3 0 \%}$ circa dell'acqua consumata per la reverse osmosis viene perso, sprecato.

Ma non basta. Solitamente, pensiamo al consumo di acqua da parte di una macchina in termini di acqua per il dialisato e per l'infusato, ma a questi vanno aggiunti quei volumi di acqua che servono al lavaggio iniziale, al risciacquo e ai lavaggi nei tempi di inattività, che costituiscono quote di cui non si ha percezione immediata. Così, un solo trattamento di HDF, per esempio, ha un consumo totale di acqua che arriva a circa $\mathbf{3 0 0}$ litri, di cui circa $\mathbf{8 0}$ sono rappresentati dall'acqua rigettata dall'osmosi.

In realtà, l'acqua rigettata dall'osmosi inversa è acqua filtrata e addolcita e, in alcuni centri, raggiunge caratteristiche biochimiche compatibili con i parametri che definiscono l'acqua potabile secondo le diverse disposizioni regionali e comunali locali. In effetti, il pre-trattamento agisce sull'acqua di rete (quella che ognuno di noi usa e beve regolarmente) e consiste in micro-filtrazione e addolcimento, che rimuovono cloramine, benzene, solventi e molti altri composti, oltre al micro-particolato in sospensione. L'acqua che esce da questo processo viene, poi, presentata alle membrane dell'osmosi inversa per la filtrazione finale. Ne deriva un'acqua che, dal

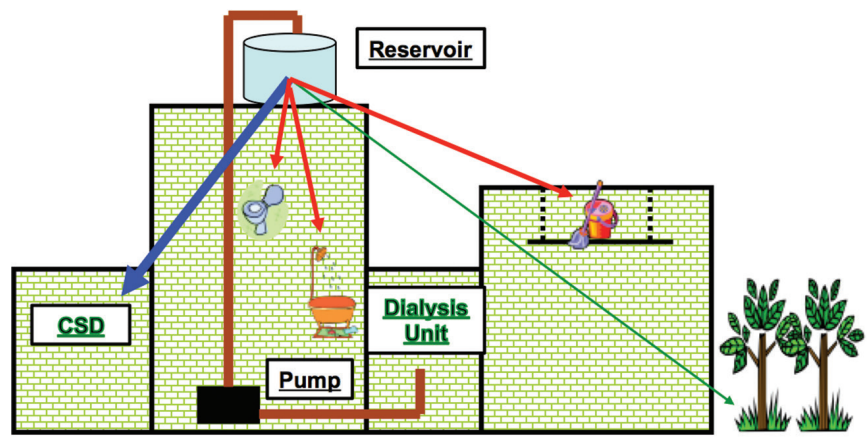

Fig. 1 - Impianto di riutilizzo dell'acqua rigettata dall'osmosi inversa. L'acqua recuperata viene pompata in una postazione alta (ultimo piano) e, da qui, per caduta, viene, poi, inviata ai diversi utilizzi prescelti: per la produzione di vapore, per le toilette, per le pulizie, per il giardino (riprodotta con autorizzazione da https://www.greendialysis.org/healthcare-professionals-and-health-authorities/water).

punto di vista chimico-fisico, è molto meglio dell'iniziale acqua di rubinetto!

$\grave{E}$, quindi, intuitivo pensare che, in un'ottica di risparmio della principale risorsa naturale, l'acqua appunto, si debba arrivare al riutilizzo dell'acqua persa. Ancora e sempre gli australiani hanno realizzato impianti di recupero dell'acqua non utilizzata che viene riutilizzata per le pulizie, per le toilette, per innaffiare le piante esterne, per la produzione di vapore a scopo pulente (4) (Fig. 1).

Altri grandi capitoli in tema di dialisi sostenibile sono quelli della produzione di rifiuti (6), che, escludendo quelli di origine biologica, sono spesso carta e materiale plastico di vario tipo. Il materiale potenzialmente infetto (il filtro, i circuiti, le siringhe usate...) è il solo a essere separato in contenitori dedicati e ammonta, secondo quanto riportato in letteratura, a 0,5-7 Kg per dialisi, sia negli USA che in Europa! Tutto il resto viene mischiato, pur essendo largamente costituito da piccoli pezzi di carta, piccoli imballaggi, confezioni plastiche..., e tutto ciò potrebbe/dovrebbe essere raccolto in contenitori dedicati, smistato e trattato nei modi più appropriati (inclusa la sterilizzazione) e, se possibile, riciclato. Le normative in essere al momento governano questi processi, sicuramente complessi, in modo, forse, non sufficientemente differenziato.

Gli imballaggi plastici sono un altro enorme problema, sia per il consumo di plastica che per i problemi di occupazione di spazio, per lo stoccaggio e per il trasporto. Sicuramente, il passaggio dalle taniche rigide alle sacche collassabili ha rappresentato un grande vantaggio, perché, a parità di volume di liquido contenuto, l'uso delle sacche consente un risparmio di circa il $30 \%$ in peso e dell' $80 \%$ in volume, con intuibili conseguenze sui trasporti, cosa che, in fondo a tutto, ha un impatto sul famoso carbonprint dialisi-relato.

\section{Possiamo cominciare a fare qualcosa?}

Le opportunità, per un centro dialisi, di migliorare la sostenibilità ambientale sarebbero tantissime e, per capire 
Tabella I - Opportunità per un centro dialisi di migliorare la sostenibilità ambientale (tradotta e abbreviata da Barraclough KA, Nephrology 2019)

\begin{tabular}{|c|c|}
\hline Illuminazione & $\begin{array}{l}\text { Conversione a lampade a basso consumo energetico; sensori di movimento; spegnere le luci, } \\
\text { se inutilizzate }\end{array}$ \\
\hline Strumentazioni elettroniche & Autoconfigurazione per la modalità di ibernazione o stand-by, spegnimento, se inutilizzate \\
\hline Riscaldamento e raffreddamento & Uso dei termostati; spegnimento, se non necessari \\
\hline Energia & Considerare l'impiego di energie rinnovabili \\
\hline Acqua & Recupero e riuso dell'acqua rigettata dall'osmosi inversa \\
\hline Rifiuti & $\begin{array}{l}\text { Differenziazione dei materiali di rifiuto; formazione dello staff sulla gestione dei rifiuti; audit periodici; } \\
\text { concordare con i fornitori il ritiro degli imballaggi; considerare il riciclo del PVC }\end{array}$ \\
\hline Approvvigionamento & Concordare con i fornitori il ritiro degli imballaggi \\
\hline Carta & Minimizzare stampe e fotocopie; stampare su 2 lati; riciclare la carta \\
\hline Trasporti & Promuovere il trasporto attivo di pazienti e staff; trasporti condivisi \\
\hline $\begin{array}{l}\text { Preparazione ai cambiamenti } \\
\text { climatici }\end{array}$ & Formare staff e pazienti alle possibili emergenze ambientali; elaborare una strategia per le emergenze \\
\hline $\begin{array}{l}\text { Piani e iniziative sul tema } \\
\text { ambientale }\end{array}$ & $\begin{array}{l}\text { Definire un Gruppo Green locale; includere la formazione alla sostenibilità ambientale nei meeting } \\
\text { periodici; fornire incentivi allo sviluppo e all'implementazione di azioni di miglioramento }\end{array}$ \\
\hline
\end{tabular}

quanto diverso sia l'approccio al problema da parte di chi ha già adottato questo obiettivo nella propria filosofia, può essere utile leggere la Tabella I tradotta da un lavoro di Barraclough KA (4), che non si fa scrupolo di ricordare, per esempio, di spegnere i computer, le luci e anche il riscaldamento, quando non utilizzati!

Nello stesso lavoro (4) vengono riportati i risultati di una survey condotta nella regione Vittoria del Sud-Est australiano, a cui hanno risposto $71 / 83$ centri dialisi, per un totale di $628 / 660$ posti dialisi. Risultava che ben il $25 \%$ dei centri era già dotato di un impianto di riciclo dell'acqua dell'osmosi inversa, che, in oltre il $40 \%$ dei centri, il personale riceve una formazione ad hoc sulla gestione dei rifiuti, che, in un terzo dei centri, si eseguono audit formali sullo stesso problema e che la sostenibilità ambientale è un parametro considerato al momento della selezione degli acquisti da effettuare.

Su questo ultimo aspetto, anche in Europa e anche in Italia si sono fatti alcuni piccoli passi avanti, avendo introdotto, nei capitolati di gara per il materiale dialitico, alcuni parametri finalizzati al principio della sostenibilità ambientale. Nella gara Consip 2016 si richiedeva di dimostrare l'ecocompatibilità di filtri e concentrati, con particolare riferimento al peso, ai materiali utilizzati e alle modalità di smaltimento. Nella gara Consip 2019, è stato introdotto il paragrafo "Criteri ambientali", identificati con la possibilità di risparmio di consumo di acqua da parte delle macchine. In una gara danese per la CRRT si richiedeva di descrivere il packaging dei prodotti in base a quanto richiesto dalla Comunità Europea, e lo stesso per le modalità di smaltimento.

\section{Progetti possibili}

Se siamo convinti tutti, e credo che lo siamo, che di queste grandi problematiche dobbiamo cominciare a interessarci, facendo anche qualche passo propositivo concreto, occorre procedere per passi successivi.

- Il primo passo è rappresentato sicuramente dalla presa di coscienza e dallo sviluppo di una piena consapevolezza dei vari aspetti della dialisi su cui lavorare. Per questo occorre documentarsi. Nella letteratura, i lavori in merito stanno crescendo, la sitografia è molto ricca e anche le industrie della dialisi hanno spesso, nel loro sito, spazi destinati all'ecocompatibilità della dialisi. Sicuramente, la conoscenza del consumo dell'acqua e il problema dei rifiuti sono i due punti da cui partire.

- L'argomento dell'ecosostenibilità potrebbe essere incluso nei meeting periodici di reparto, a fini di diffusione di conoscenze e di valutazione di progetti migliorativi e a scopo di audit.

- Molto utile sarebbe, come suggerito dagli inglesi e dagli australiani $(2,4)$, la costituzione, all'interno di ciascun centro dialisi, di un gruppo green, a composizione mista, che si formi, per esempio, sulla gestione dei rifiuti, che si interfacci con le figure preposte all'argomento in Direzione e che, poi, si incarichi di diffondere le conoscenze a tutti i medici e infermieri. Si potrebbero proporre dei progetti di miglioramento e si potrebbero chiedere degli incentivi per le figure identificate come responsabili.

- Si potrebbero eseguire, poi, degli audit periodici su temi identificati come meritevoli, di facile comprensione da parte di tutti e con possibilità di semplici azioni concrete al riguardo (per esempio, limitare l'uso della carta a scopo di scrittura o di fotocopie o di stampe varie, promuovere I'utilizzo di carta riciclata, limitare I'uso della luce o del riscaldamento in ambienti non utilizzati costantemente). 


\section{Innovazioni tecnologiche}

La modifica strutturale degli impianti di trattamento e distribuzione dell'acqua per dialisi al fine di recuperare e, successivamente, riciclare l'acqua rigettata dell'osmosi è complessa e richiede investimenti che non avrebbero il tempo utile per essere ammortizzati. Viceversa, per i centri che devono essere costruiti o per i centri che devono rinnovare l'impianto idrico, si dovrebbe cominciare a considerare la costruzione di sistemi di recupero dell'acqua. Ovviamente, questo prevede che vengano sensibilizzate da molto tempo prima la Direzione del proprio ospedale e l'Ingegneria Clinica, fornendo spiegazioni che giustifichino l'inevitabile aumento di spesa costruttiva. II riciclo dell'acqua per altri scopi può parzialmente compensare la maggiore spesa.

Un'innovazione più fattibile, che va verso il principio di una dialisi sostenibile, è sicuramente il sistema centralizzato di distribuzione del concentrato. In altre parole, il concentrato acido è presente solo nella centrale, all'interno di un grande contenitore, e da qui viene inviato, con tubature dedicate, a tutte le postazioni dialitiche, dove, poi, verrà miscelato con il bicarbonato. In Italia, già parecchi centri dialisi hanno adottato questo tipo di soluzione. Un'alternativa ulteriore è la preparazione centralizzata di un bagno dialisi già completo, che viene erogato in quanto tale alle macchine (7).

I vantaggi diretti e indiretti sono molti:

- Riduzione del tempo di preparazione della macchina

- Riduzione della plastica e del materiale di rifiuto

- Nessuno spreco delle soluzioni concentrate

- Riduzione degli spazi necessari allo stoccaggio

- Riduzione del carico di lavoro (sollevamento) per il personale

- Riduzione del trasporto merci (riduzione emissioni)

Ci sono, però, anche dei limiti. Uno è la difficoltà di individualizzare la composizione del bagno dialisi, soprattutto in termini di composizione elettrolitica. Un altro è, per la distribuzione centralizzata del bagno dialisi, il rischio di contaminazione microbiologica legato al bicarbonato; rischio non presente, invece, nel caso di distribuzione centralizzata del solo concentrato acido, proprio per il $\mathrm{pH}$ estremamente basso della soluzione.

$\mathrm{E}$, infine, non possiamo non citare l'ipotesi di ritornare alle resine, già usate decenni fa, con parecchi problemi che le portarono in disuso e che le più recenti tecnologie potrebbero, invece, superare. I sorbenti verrebbero impiegati per rigenerare parte del dialisato esausto, garantendo, così, un notevole risparmio di acqua. Sistemi di questo genere potrebbero risultare di grande ausilio nelle aree geografiche aride del continente o in zone rurali o remote.

\section{Conclusione}

Il tempo per un ripensamento radicale della modalità di erogazione della terapia dialitica in funzione del rispetto dell'ambiente è sicuramente già arrivato (8). Anche ERAEDTA, negli ultimi anni, ha dato avvio a un ampio numero di iniziative finalizzate a "rendere verde" il settore sanitario renale in ambito UE (9).

Prima di pensare a grandi innovazioni tecnologiche, noi tutti dobbiamo scrollarci di dosso abitudini inveterate e rivedere criticamente le nostre attività sul lavoro con un'ottica "green". Un piano strutturato per il semplice risparmio della carta e il suo riutilizzo prima di essere gettata negli appositi spazi o il controllo nell'utilizzo dell'illuminazione ambientale anche sul posto di lavoro potrebbero essere dei semplicissimi punti di partenza. Le esperienze consolidate di altri centri possono servire da guida per gli ulteriori passi.

La costruzione dei nuovi centri dialisi non può più prescindere da quanto è già stato realizzato altrove, sia in termini strutturali che di consumi energetici. Una nuova gestione dell'acqua per dialisi diventa un capitolo obbligatorio nei piani di ogni nuova progettazione.

\section{Disclosures}

Conflict of interest: The authors declare no conflict of interest. Financial support: This research received no specific grant from any funding agency in the public, commercial, or not-for-profit sectors. progettazione.

\section{Bibliografia}

1. Sustainability Series: Snapshot of Kidney Care. Examining the environmental impact of kidney care. https://sustainablehealthcare.org.uk/blog/sustainability-series-snapshot-kidneycare.

2. Connor A, Lillywhite R, Cooke MW. The Carbon Footprint of a Renal Service in the United Kingdom. QJM. 2010;103:965-975.

3. Lim AEK, Perkins A, Agar JWM. The carbon footprint of an Australian satellite hemodialysis unit. Aust Health Rev. 2013; 37(3):369-74. doi: 10.1071/AH13022.

4. Barraclough KA, Gleeson A, Holt SG, Agar JWM. Green dialysis survey: establishing baseline for environmental sustainability across dialysis facilities in Victoria, Australia. Nephrology. 2019;24:88-93.

5. Sustainability the seventh dimension of quality. Working for Better Kidney Care. http://renaltsar.blogspot.com/2010/05/ sustainability-seventh-dimension-of.html.

6. Piccoli GB, Nazha M, Ferraresi M, Vigotti FN, Pereno A, Barbero $S$. Eco-dialysis: the financial and economical cost of dialysis waste products: is a "cradle to cradle" model feasible for planet-friendly hemodialysis waste management? Nephrol Dial Transplant. 2015;30:1018-27. doi: 10.1093/ndt/gfv031.

7. Kawanishi H. The central dialysis fluid delivery system (CDDS): is it specialty in Japan? Ren Replace Ther. 2016;2:1. https://doi. org/10.1186/s41100-016-0016-4.

8. Bendine G, Autin F, Fabre B, et al. Hemodialysis therapy and sustainable growth: a corporate experience in France. Nephrol Dial Transplant. 2020;1-7. doi: 10.1093/ndt/gfz284.

9. Blankestijn PJ, Arici M, Bruchfeld A, et al. ERA-EDTA invests in transformation to greener health care. Nephrol Dial Transplant. 2018;33(6):901-3. 